\title{
Computed Tomography Images under Deep Learning Algorithm in the Diagnosis of Perioperative Rehabilitation Nursing for Patients with Lung Cancer
}

\author{
Sha Yan $\mathbb{D}^{D},{ }^{1}$ Qin Huang $\mathbb{D}^{1},{ }^{1}$ Siying Yu $\mathbb{D}^{1},{ }^{1}$ and Zhenxing Liu $\mathbb{D}^{2}$ \\ ${ }^{1}$ Department of Operating Room, No. 4 Hospital of Wuhan, Wuhan, Hubei 430000, China \\ ${ }^{2}$ Department of Social Services, No. 4 Hospital of Wuhan, Wuhan, Hubei 430000, China \\ Correspondence should be addressed to Zhenxing Liu; liuzhenxing@st.btbu.edu.cn
}

Received 30 August 2021; Revised 22 December 2021; Accepted 8 January 2022; Published 25 January 2022

Academic Editor: Gustavo Ramirez

Copyright (C) 2022 Sha Yan et al. This is an open access article distributed under the Creative Commons Attribution License, which permits unrestricted use, distribution, and reproduction in any medium, provided the original work is properly cited.

\begin{abstract}
This study aim was to explore the application effect of computed tomography (CT) image segmentation based on deep learning algorithm in the diagnosis of lung cancer. In this study, a two-dimensional (2D) convolutional neural network (CNN) and threedimensional (3D) CNN fusion model was constructed firstly. Subsequently, 60 patients with lung cancer were randomly divided into a control group and an intervention group to receive perioperative routine nursing and rehabilitation nursing, respectively. The results revealed that the Dice value (0.876), sensitivity (0.849), and positive predictive value (PPV) (0.875) of the hybrid feature fusion model (HFFM) constructed in this study for lung cancer CT image segmentation were higher than those of other models, and the accuracy rate for lung cancer diagnosis was $96.7 \%$. After nursing intervention, the partial arterial oxygen pressure $\left(\mathrm{PaO}_{2}\right)$ and partial arterial carbon dioxide pressure $\left(\mathrm{PaCO}_{2}\right)$ in the control group were $80.54 \mathrm{mmHg}$ and $39.81 \mathrm{mmHg}$, respectively, while those in the intervention group were $83.09 \mathrm{mmHg}$ and $36.75 \mathrm{mmHg}$, respectively. After intervention, the maximal voluntary ventilation (MVV) \%, forced vital capacity (FVC) \%, and forced expiratory volume in 1 sec (FEV1) \%) in the intervention group were $76.03 \%, 82.14 \%$, and $89.76 \%$, respectively. It suggested that compared with the control group, the pulmonary function indexes of the intervention group improved significantly after nursing intervention $(P<0.05)$. In summary, the HFFM constructed in this study can be used for segmentation and classification of CT images of lung cancer patients, which can improve the accuracy of diagnosis and help improve the lung function and quality of life of patients.
\end{abstract}

\section{Introduction}

Lung cancer is the most common malignant tumor (i.e., the main malignant tumor) that causes human deaths, and its incidence is increasing year by year [1-3]. Screening for lung nodules is an effective way to prevent lung cancer [4].

In the diagnosis and treatment of clinical diseases, imaging examinations can provide the main reference materials for the diagnosis of lung diseases. In the examination of lung diseases using imaging techniques, CT images occupy an important and local position $[5,6]$. The era of artificial intelligence has come, and the development of deep learning technology has given birth to various developments. In this context, some scholars have proposed imaging omics to extract high-throughput omics features from cancer medical images. The features that doctors can see from the image data with naked eyes, such as shape features, tumor size, and wavelet transform, can have a good predictive effect through deep learning technology modeling. Imaging is achieved by combining image features with clinical information to mine data to determine image markers $[7,8]$. With the increase in the incidence of lung cancer, the CT image information of patients also shows an exponential growth trend, so it is very important to accurately identify lung nodules from CT images $[9,10]$. Deep learning can realize image translation and other operations through weight sharing and other methods, optimize the complex operations of image preprocessing, and realize target detection and image classification [11]. Medical image analysis is greatly affected by subjective experience. The use of computer technology (CT) 


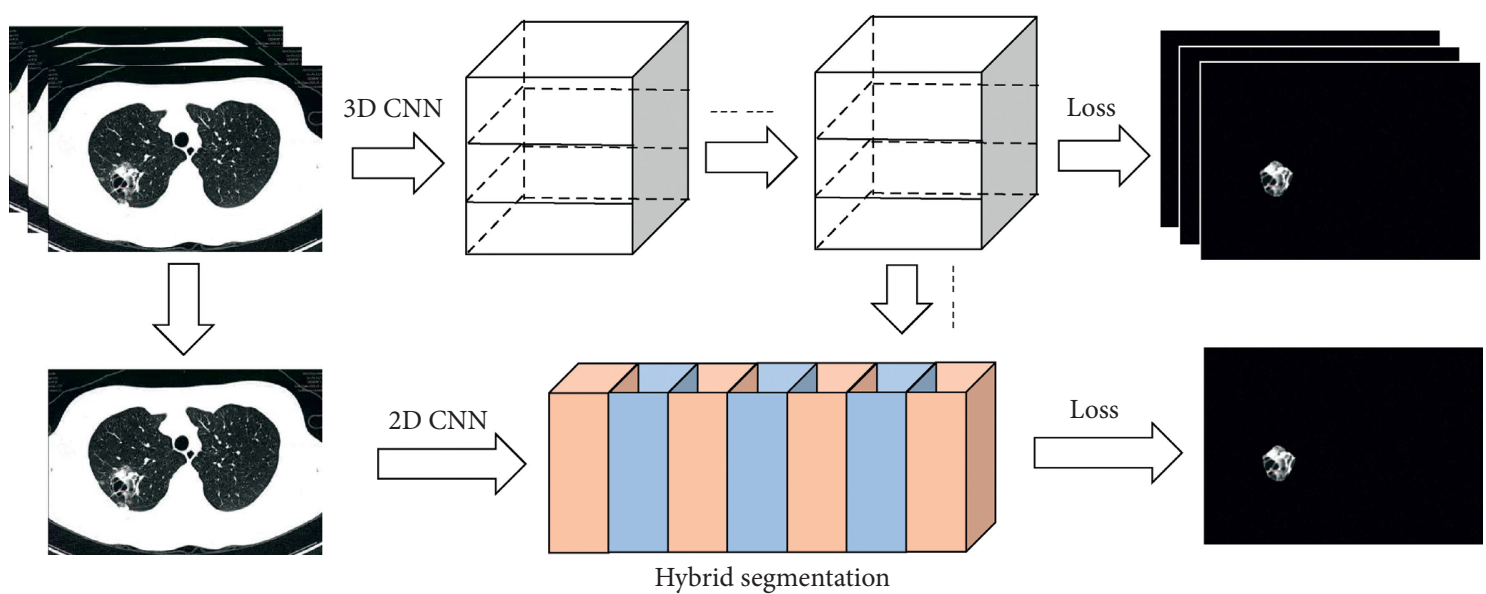

FIgURE 1: The basic structure of the hybrid segmentation network.

for auxiliary diagnosis of diseases can quickly and accurately realize disease judgments and reduce the missed diagnosis rate and misdiagnosis rate of clinical diagnosis [12].

In order to improve the diagnosis rate of clinical lung cancer and improve the quality of life of patients after surgery, a deep learning model for lung cancer CT image segmentation was proposed and applied to the clinical diagnosis of lung cancer. Then, the impacts of nursing intervention during the perioperative period on the pulmonary function and quality of life of patients after lobectomy were analyzed. The results of this study were intended to provide a reference for improving the clinical diagnosis and treatment of lung cancer.

\section{Methods}

2.1. CT Segmentation of Lung Cancer Based on Deep Learning. The current methods used for medical image segmentation processing are mainly divided into techniques based on manual features and depth features [13]. The convolutional neural network (CNN) model has been widely used in image classification and segmentation and it is mainly based on the image block training model to classify the central pixel [14]. However, the CNN model has certain limitations. The features in the model are only limited to image blocks, while ignoring the global features. In the field of medical images such as CT, images have $3 \mathrm{D}$ data characteristics compared with natural images. Since the current learning effect for different slices is low, the correlation between each slice cannot be effectively obtained. Therefore, a hybrid segmentation network based on the CNN model was constructed for lung cancer segmentation of 3D CT images.

2.1.1. The Overall Structure of the Hybrid Segmentation Network. The basic structure of the hybrid segmentation network constructed in this study is shown in Figure 1. The model was mainly composed of a $3 \mathrm{D} \mathrm{CNN}$ and a $2 \mathrm{D} \mathrm{CNN}$. Among them, 3D CNN can be used to learn 3D information, and $2 \mathrm{D} \mathrm{CNN}$ was used to obtain detailed semantic information. Using the hybrid feature fusion model (HFFM), the features extracted by $3 \mathrm{D} \mathrm{CNN}$ and $2 \mathrm{D} \mathrm{CNN}$ can be effectively fused. The final loss value obtained by the model was also divided into $2 \mathrm{D}$ and $3 \mathrm{D}$ parts, which were used for network optimization training.

2.1.2. Design of 3D CNN Model. The basic structure of the $3 \mathrm{D}$ CNN model designed in this study is shown in Figure 2. The model structure was very similar to the $3 \mathrm{D}$ U-Net structure, in which the encoder can be used to extract image features and perform image segmentation. Some improvements were made on the basis of 3D U-Net. Firstly, the $3 \times 3 \times 3$ convolution in the original model was replaced with the multiscale separable convolution (MSC) module [15]. Secondly, a separable spatial/temporal 3D (S3D) [16] was adopted to replace the pooling operation to reduce the size of the feature map. Finally, the upsampling feature map was cascaded with the feature map generated by the encoder, and the MSC module was used to adjust the number of feature maps. Through the above improvements, the parameter setting and calculation cost of the original 3D U-Net can be reduced.

2.1.3. Design of $2 D C N N$. In the classic CNN model, the resolution of feature maps can be greatly reduced through continuous convolution operations or stride convolution operations. Feature maps with abstract features and low resolution can improve the performance of model classification but cannot effectively perform the semantic segmentation. Therefore, the $2 \mathrm{D} \mathrm{CNN}$ designed in this study adopted the dilated convolution structure, replacing part of the convolution structure with a dilated unit block (DUB) structure containing two residual convolutions connected by holes. The basic structure of the model is shown in Figure 3.

In the empty convolution operation, it had to specify a value $x[i]$ and a convolution kernel $w[k]$, and based on the corresponding empty rate $r$, the final calculation formula of the empty convolution feature map $y[i]$ could be expressed as follows: 

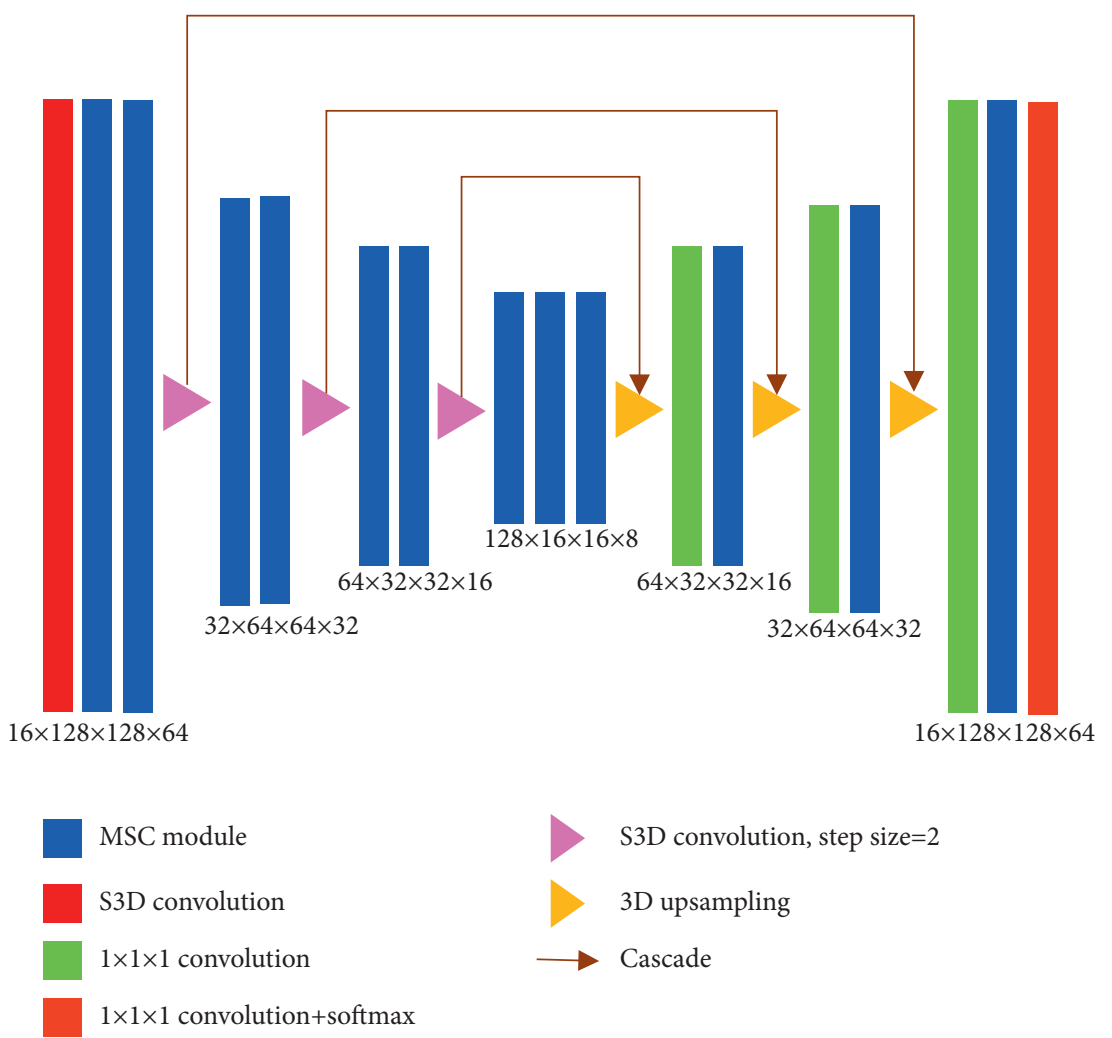

Figure 2: The basic structure of the 3D CNN model.

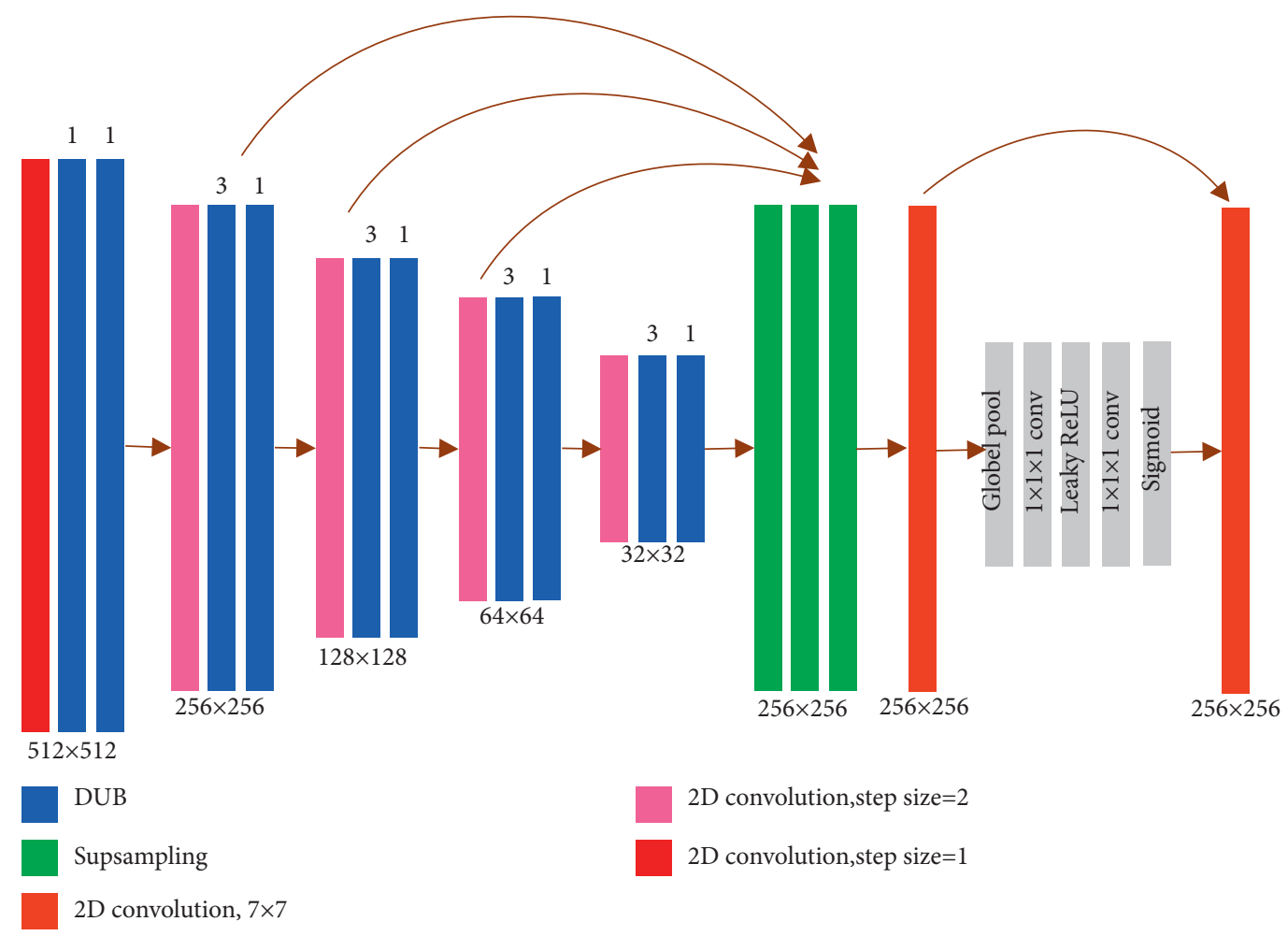

Figure 3: The basic structure of the designed 2D CNN model. 


$$
y[i]=\sum_{k} x[i+r k] w[k] .
$$

The size of the input image of the model was $512 \times 512$, and the image was decomposed into 16 feature maps using a convolutional layer of $3 \times 3 \times 3$ structure; and features in the feature map were extracted using DUB and stride convolution until the size of the feature map was $32 \times 32$. The feature maps were upsampled to expand the image size to $256 \times 256$ for cascading. After the cascading operation, the squeeze-and-excitation (SE) and extrusion operations were adopted to build the dependency between the feature maps; and the $7 \times 7$ convolution kernel was used to replace the original $3 \times 3$ convolution kernel to increase the image segmentation effect of the model.

2.1.4. Design of HFFM. In order to be able to fuse the feature maps generated by the $3 \mathrm{D} \mathrm{CNN}$ module with the feature maps generated by the 2D CNN module, a hybrid features fusion module (HFFM) was constructed in this study to perform the fusion of the above feature maps.

It was assumed that the feature map before the last layer of the convolutional layer in the $3 \mathrm{D}$ CNN model was upsampled to obtain a feature map $x_{3 D} \in R^{n \times c 1 \times 256 \times 256 \times 64}$ of $256 \times 256 \times 64$, in which $n$ referred to the batch size and $c 1$ was the number of channels. $x_{2}{ }_{D} \in R^{m \times c 2 \times 256 \times 256}$ was determined, in which $m$ was the batch size and $c 2$ referred to the number of channels. Finally, the batch size in the model was set to 1 , and finally it was $x_{3} \in R^{c 1 \times 256 \times 256 \times 64}$. The $2 \mathrm{D} \mathrm{CNN}$ module mainly sliced CT images along the $z$-axis. To fuse the feature maps output by the two CNN models, it was necessary to use the slices in the $2 \mathrm{D}$ CNN model to perform cropping in the 3D CNN model to obtain the index of the CT slices, so the size of the picture is set to $x_{3 D}^{\prime} \epsilon R^{n \times c 1 \times 256 \times 256}$. The mathematical expression of the cascade operation was given as follows:

$$
U=\operatorname{Cat}\left(x_{3 d}^{\prime}, x_{2 d}^{\prime}\right), \quad U \in R^{m \times(c 1+c 2) \times 256 \times 256} .
$$

The cascaded feature map can integrate the advantages of the two models and allow simultaneous training. After fusion, a convolutional layer had to be added for optimization processing, the feature map size was restored through an upsampling operation, and finally the convolutional layer and the softmax layer were used to segment the image.

2.2. Research Objects. The patients with lung cancer who were in hospital from June 2018 to December 2020 were selected as the research objects. The inclusion criteria were defined as follows: patients with clinical diagnosis of lung cancer; patients who performed with CT scan; and patients who underwent lobectomy under general anesthesia or combined lymphadenectomy for surgical treatment. A pulmonary function examination was required on admission for all patients, and maximal voluntary ventilation (MVV) $\%>55 \%$ and forced expiratory volume in $1 \mathrm{sec}$ $(\mathrm{FEV} 1)>2 \mathrm{~L}$ should be satisfied. The exclusion criteria were defined as follows: those who suffered from diseases that affect pulmonary function such as asthma and respiratory failure; those who were malnourished or obese; patients with cardiovascular and cerebrovascular diseases; those with organic diseases or organ dysfunction; those who cannot be treated with mechanical ventilation after surgery; and those who receive chemotherapy or radiotherapy before surgery.

Cases were screened according to the inclusion and exclusion criteria, and finally a total of 60 patients were included in this study. According to the random number table method, the patients were randomly divided into an intervention group and a control group, with 30 patients in each group. This study had been approved by the ethics committee of hospital, and all patients had signed the informed consent forms.

2.3. CT Scan. The dual-source CT was adopted to scan the patient's chest. The scan parameters were set as follows: voltage of $100 \mathrm{kv}$, current of $120 \mathrm{~mA}$, pitch of 0.75 , layer thickness of $5 \mathrm{~mm}$, and the layer thickness of the diseased area of $1.00 \mathrm{~mm}$. CT scan was required for all cases, and the width of the chest plain scan window was 1500 1600 HU. The window level was $-700 \sim-600 \mathrm{HU}$, the window width of the mediastinal window was $350 \sim 400 \mathrm{HU}$, and the window level was $40 \mathrm{HU}$. The CT images obtained by scanning were transmitted to the workstation, and the lesions and signs of the images were analyzed.

2.4. Nursing Methods of Different Groups. For patients in control group, they were advised to quit smoking before surgery, breathing training was performed 2 days before surgery, enema cleaning was performed 1 day before surgery, and patients were given appropriate psychological nursing. Routine oxygen inhalation should be given after the surgery, and the changes of the patient's physiological indicators should be observed. The self-controlled epidural analgesics was adopted for pain control, and aerosol inhalation was given to prevent atelectasis. The patients were encouraged to get out of bed to help gastrointestinal peristalsis.

For patients in the intervention group, they were given regular nursing similar to that for patients in the control group; they received preoperative psychological nursing to stabilize their emotions and explain the knowledge related to surgery to patients; they were performed the preoperative respiratory function training after admission to 2 days before the surgery (the training content included abdominal breathing, lip-reducing breathing, and effective cough training); and they received the following postoperative rehabilitation intervention: rehabilitation intervention was performed after the patient is awake; the intervention content included posture guidance, respiratory function training intervention, limb function training, and getting out of bed.

\subsection{Observation Indicators}

2.5.1. Evaluation of CT Image Segmentation Performance. The Dice, sensitivity, and positive predictive value were selected to evaluate the effect of CT image segmentation. 
Dice was used to evaluate the degree of coincidence between the segmented image and the gold standard [17]. Sensitivity value was used to evaluate how many positive patients in the segmented sample were correctly classified. The positive predictive value was used to evaluate the true positive cases in the segmentation classification as positive cases. The calculation equations for different indicators were given as follows:

$$
\begin{aligned}
\text { Dice } & =\frac{2 \mathrm{TP}}{\mathrm{FP}+2 \mathrm{TP}+\mathrm{FN}}, \\
\text { Sensitivity } & =\frac{\mathrm{TP}}{\mathrm{TP}+\mathrm{FN}}, \\
\mathrm{PPV} & =\frac{\mathrm{TP}}{\mathrm{TP}+\mathrm{FP}} .
\end{aligned}
$$

In the above equations, $\mathrm{TP}$ was the number of true positives, FP referred to the number of false positives, and FN represented the number of false negatives.

2.5.2. Evaluation of Patient Rehabilitation Effect. The clinical data of all patients were collected. The preoperative data included the patient's age, gender, and smoking history. Postoperative data included postoperative $24 \mathrm{~h}$ thoracic drainage, total drainage, extubation time, complication rate, and total hospital stay.

The pulmonary function indexes were measured. The MVV, FVC, and FEV1 of patients were evaluated by pulmonary function test at the time of admission and 7 days after surgery, respectively. MVV was the maximum volume of air inhaled or exhaled per minute after deep breathing, which can feed back the total volume of ventilation per unit time. When the MVV\% was greater than $80 \%$, it meant that the patient's ventilatory function was normal; there was a slight decrease in ventilatory function when MVV\% was $60-79 \%$, there was a decrease in ventilatory function when MVV\% was 40-59\%, and there was severely decreased ventilatory function when MVV\% was less than 39\%.

The patient's blood gas index (partial arterial oxygen pressure $\left(\mathrm{PaO}_{2}\right)$ and partial arterial carbon dioxide pressure $\left(\mathrm{PaCO}_{2}\right)$ ) were detected on admission and 7 days after surgery. When the $\mathrm{PaO}_{2}$ value was less than $55 \mathrm{mmHg}$, respiratory failure was considered, and the normal range of $\mathrm{PaCO}_{2}$ was $35 \sim 45 \mathrm{mmHg}$.

The SF-36 scale was used to evaluate the quality of life of patients. The scale included 8 dimensions: physiological function, physiological intelligence, trunk pain, health status, energy, social function, emotional function, and mental health. The total score of the scale was 100 points. The higher the patient's score, the better the health status, and the higher the quality of life.

2.6. Statistical Analysis. The SPSS19.0 statistical software package was adopted for statistical processing and analysis of experimental data. The count data was expressed as frequency $[n(\%)]$, and the chi-square test was used for comparison. The measurement data was expressed in the form of mean \pm standard deviation $(\bar{x} \pm s)$, and the independent sample $t$-test and repeated measures analysis of variance were used for comparison. $P<0.05$ indicated that the difference was statistically significant.

\section{Results and Analysis}

3.1. Test of CT Image Segmentation Model. The CT images of 60 patients were collected, and the sample size was expanded through rotation, resolution adjustment, and other methods, and finally 150 sample data were obtained. The data samples were randomly divided into a training set and a testing set, including 20 and 130 sample data in each set, respectively. The PyTorch deep learning framework was selected to write the code, the Adam function was adopted to optimize the training, the initial learning rate was set to 0.0001 , the loss function was the GDL function, and the activation function was the LeakyReLU function.

Firstly, Dice, sensitivity, and PPV were selected to perform quantitative evaluation of $2 \mathrm{D}$ CNN [18], 3D CNN [19], U-Net [20], and the model proposed in this paper. The results is shown in Figure 4. The accuracy of 2D CNN, 3D $\mathrm{CNN}$, and U-Net was $80.0 \%, 85.0 \%$, and $91.7 \%$, respectively. It can be seen that the segmentation performance of the $2 \mathrm{D}$ $\mathrm{CNN}$ model was relatively poor, followed by the U-Net model; and the HFFM proposed in this study for CT image segmentation showed the highest Dice value (0.876), sensitivity (0.849), and PPV (0.875). In addition, after CT image segmentation based on the model proposed, it was used for the auxiliary diagnosis of lung cancer, and the diagnosis rate was as high as $96.7 \%$.

The manual segmentation results were undertaken as the gold standard to subjectively evaluate the differences in the effect of different models on patient CT image tumor segmentation, and the results is shown in Figure 5. It illustrated that the image segmented by the method proposed was closer to the gold standard. The segmentation results of the 2D CNN model differed the most from the gold standard. In addition, the segmentation effect of the U-Net model was better, but the segmentation effect for early inconspicuous lesions was poor. The red and blue circles in the figure were marks for feature extraction.

3.2. Basic Data of Patients. The differences in the clinical data of patients in the control group and the intervention group were compared, and the results are shown in Table 1. There was no statistical difference in the average age, body mass index (BMI), smoking history, gender ratio, cancer stage, and histological type of patients in the two groups $(P>0.05)$. It showed that the general information of the two groups of patients was relatively balanced, and the results were comparable.

3.3. The Effect of Perioperative Nursing Intervention on Pulmonary Function of Patients. The changes in the respiratory function of patients after lung resection were the most obvious, especially the decrease in lung volume and MVV, in addition to the increase in residual air volume and the 


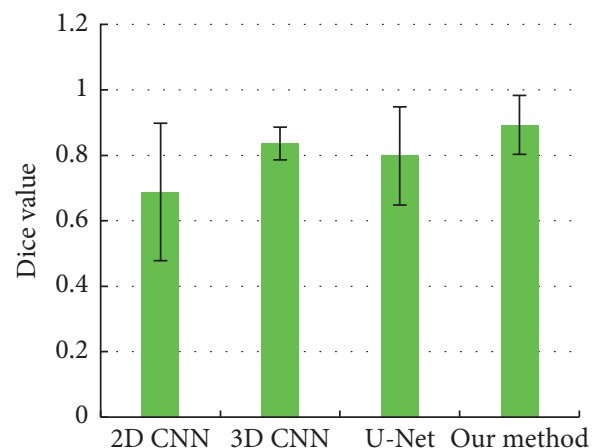

(a)

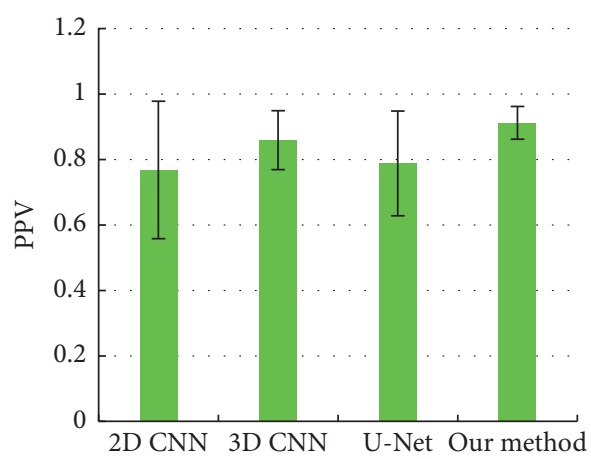

(c)

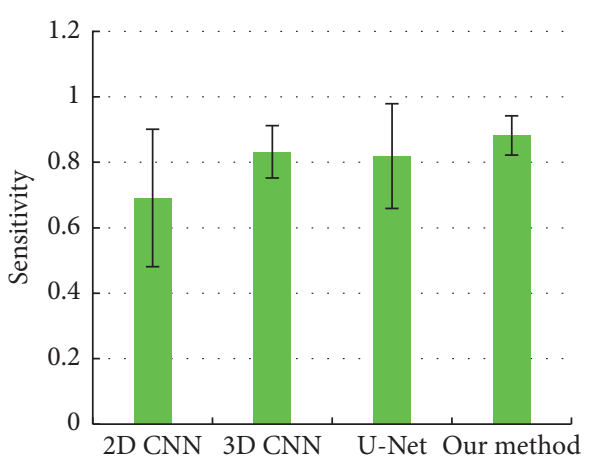

(b)

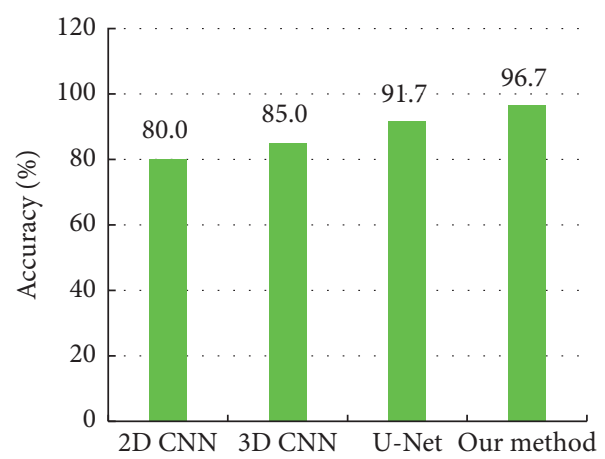

(d)

FIGURE 4: Quantitative evaluation of CT image segmentation efficiency of different models. (a-d) The results of Dice, sensitivity, PPV, and diagnosis accuracy, respectively.

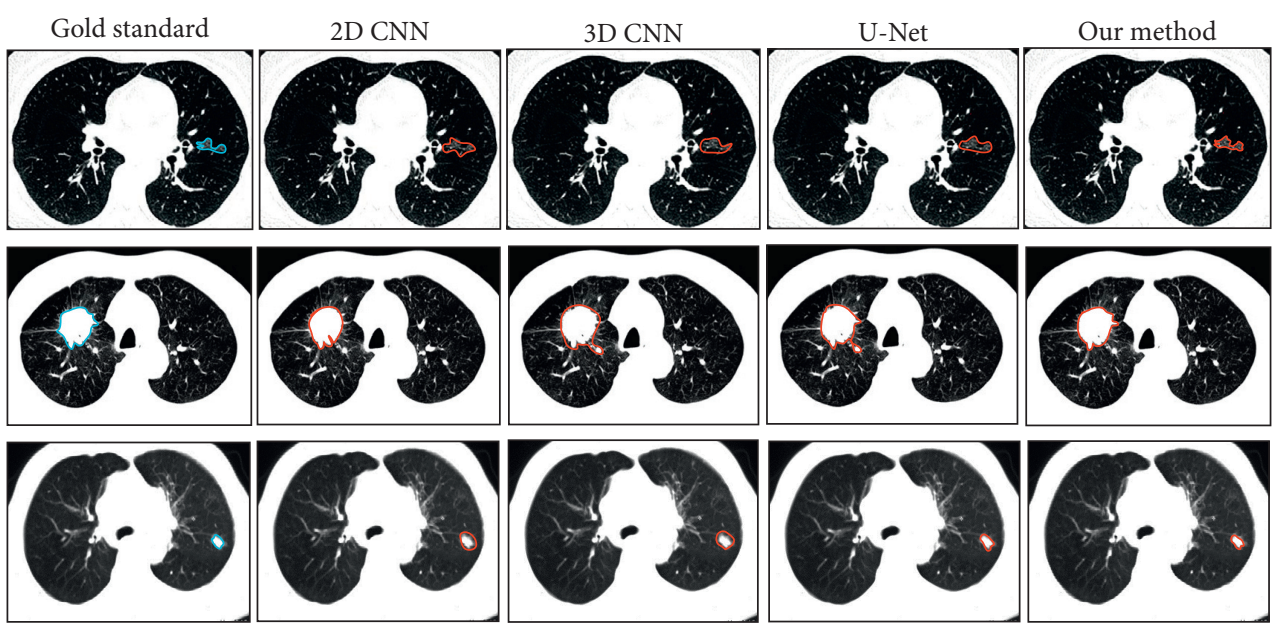

FIGURE 5: Subjective evaluation of CT image segmentation efficiency of different models.

decrease in oxygen utilization coefficient, which increased the probability of patients with hypoxia. In this comparison, the differences in the MVV\%, FVC\%, and FEV1\% of the patients' pulmonary function indexes before and after treatment were analyzed and compared, and the results is shown in Figure 6. The MVV\%, FVC\%, and FEV1\% of the intervention group after the intervention were $76.03 \%, 82.14 \%$, and $89.76 \%$, respectively, while those in the control group were $68.62 \%, 78.87 \%$, and $76.36 \%$, respectively.
It indicated that there was no statistically obvious difference in the $\mathrm{MVV} \%, \mathrm{FVC} \%$, and $\mathrm{FEV} 1 \%$ for patients between the control group and intervention group before treatment $(P>0.05)$. After treatment, the pulmonary function indexes of the two groups of patients had changed to different degrees. Compared with the control group, the MVV\%, FVC\%, and FEV1\% values of the pulmonary function indexes of the intervention group patients were dramatically increased, and the differences were statistically significant $(P<0.05)$. 
TABLE 1: Comparison of general information of the two groups of patients.

\begin{tabular}{lcrr}
\hline Item & Control group $(n=30)$ & Intervention group $(n=30)$ & Value $\left(t / \chi^{2}\right)$ \\
\hline Age (years old) & $61.05 \pm 5.06$ & $60.28 \pm 2.11$ & 0.663 \\
Males $(n / \%)$ & $21 / 70.0$ & $20 / 66.7$ & 0.698 \\
BMI $\left(\mathrm{kg} / \mathrm{m}^{2}\right)$ & $22.37 \pm 1.29$ & $22.18 \pm 1.97$ & 0.512 \\
Smoking history (years) & $12.65 \pm 4.23$ & $11.39 \pm 5.27$ & 0.716 \\
Cancer stage $(n / \%)$ & & & 0.583 \\
Stage I & $6 / 20.0$ & $5 / 16.7$ & 0.845 \\
Stage II & $18 / 60.0$ & $19 / 63.3$ & 0.926 \\
Stage III & $6 / 20.0$ & $6 / 20.0$ & 0.617 \\
Histological type $(n / \%)$ & & & \\
Squamous cell carcinoma & $20 / 66.7$ & $19 / 63.3$ & 0.533 \\
Adenocarcinoma & $9 / 30.0$ & $9 / 30.0$ & 0.518 \\
Small cell carcinoma & $1 / 3.3$ & $2 / 6.7$ & \\
\hline
\end{tabular}

Note: BMI refers to body mass index.

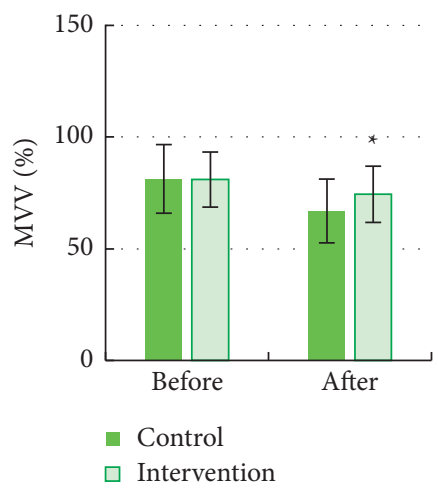

(a)

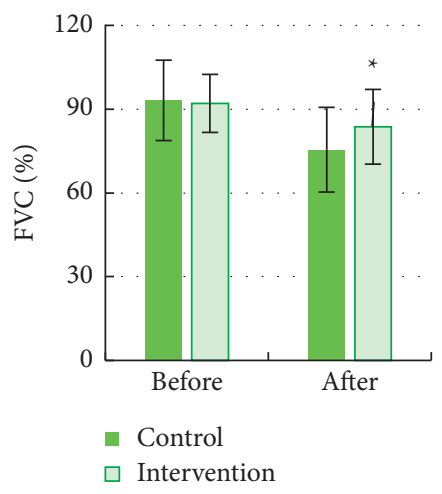

(b)

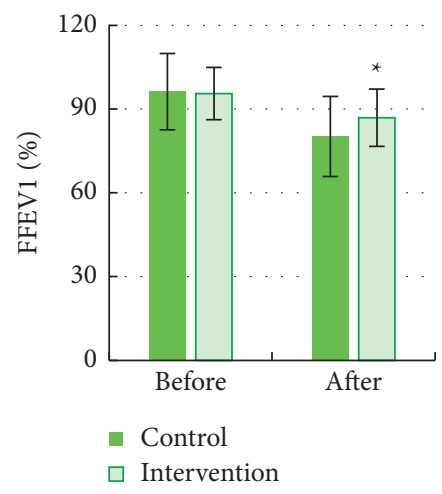

(c)

FIGURE 6: Comparison of changes in pulmonary function indexes between the two groups of patients before and after nursing intervention. (a) Comparison of MVV\%; (b) FVC\% comparison results; and (c) the comparison result of FEV1\%. *The difference was statistically obvious in contrast to the control group $(P<0.05)$.

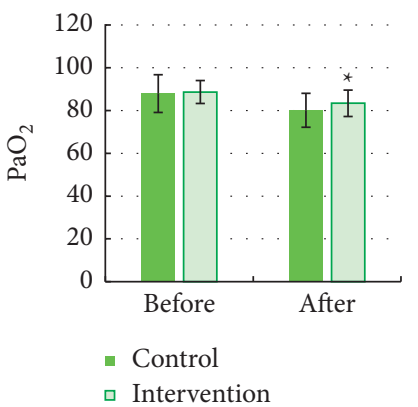

(a)

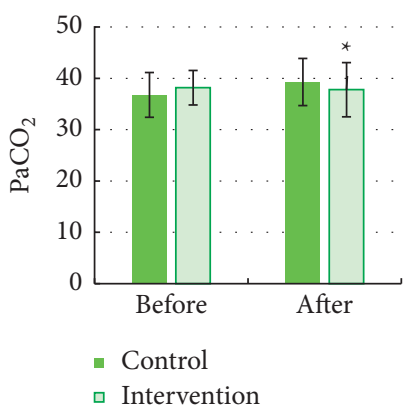

(b)

FIGURE 7: Comparison of the changes in blood gas indexes between the two groups of patients before and after nursing intervention. (a) The change of $\mathrm{PaO}_{2}$ and (b) the change of $\mathrm{PaCO}_{2}$. ${ }^{*}$ The difference was statistically obvious in contrast to the control group $(P<0.05)$.

The improvement of pulmonary function was due to rehabilitation nursing interventions such as patient's breathing training, limb activity training, and postoperative walking ability exercise during the perioperative period. The above operation can effectively improve the patient's pulmonary ventilation function, promote the absorption rate of oxygen by the alveolar capillaries, and then enhance the recovery effect of the pulmonary function [21].
3.4. The Influence of Perioperative Nursing Intervention on Blood Gas Indexes of Patients. The differences in blood gas index $\mathrm{PaO}_{2}$ and $\mathrm{PaCO}_{2}$ of patients before and after treatment were compared, and the results are shown in Figure 7. Pursed-lip breathing training can reduce the patient's exhalation speed, increase the pressure inside the airway, and facilitate the emptying of air inside the alveoli [22]. The decrease in respiratory rate can increase the exchange rate 


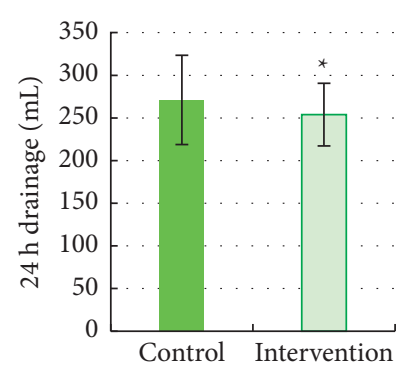

(a)

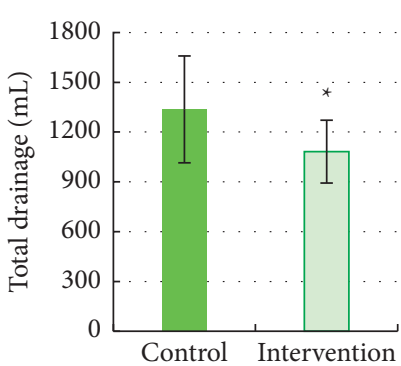

(b)

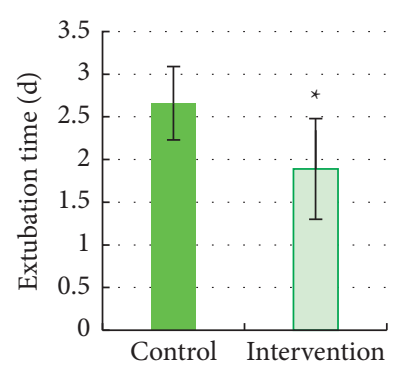

(c)

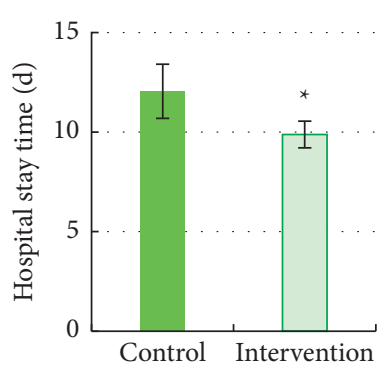

(d)

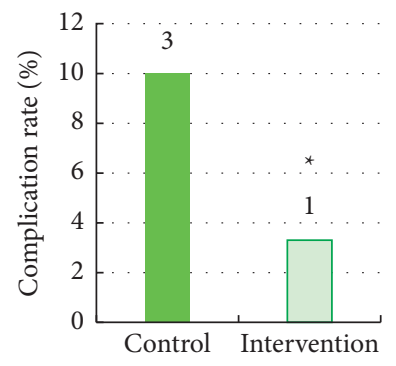

(e)

Figure 8: Comparison of differences in surgical-related indicators between the two groups of patients after nursing intervention. (a-e) The changes in $24 \mathrm{~h}$ drainage, total drainage, chest tube extraction time, hospital stay, and complication rate, respectively. ${ }^{*}$ The difference was statistically obvious in contrast to the control group $(P<0.05)$.

and diffusion of gas, increase the absorption rate of oxygen by the alveoli, and effectively avoid postoperative hypoxemia [23]. The results of this study showed that there was no visible difference in blood gas indexes $\mathrm{PaO}_{2}$ and $\mathrm{PaCO}_{2}$ between the control group and intervention group patients before treatment $(P>0.05)$. After nursing intervention, the partial arterial oxygen pressure $\left(\mathrm{PaO}_{2}\right)$ and partial arterial carbon dioxide pressure $\left(\mathrm{PaCO}_{2}\right)$ in the control group were $80.54 \mathrm{mmHg}$ and $39.81 \mathrm{mmHg}$, respectively, while those in the intervention group were $83.09 \mathrm{mmHg}$ and $36.75 \mathrm{mmHg}$, respectively.

The blood gas index of the two groups of patients also changed after treatment. Among them, compared with the control group, the $\mathrm{PaO}_{2}$ value of intervention group patients increased greatly, and the $\mathrm{PaCO}_{2}$ value decreased dramatically, showing statistically obvious differences $(P<0.05)$.

\subsection{The Impacts of Perioperative Nursing Intervention on} Operation-Related Indexes of Patients. The differences in $24 \mathrm{~h}$ drainage, total drainage, chest tube extraction time, hospital stay, and complication rates of surgical-related indicators after intervention between the two groups of patients were compared. As shown in Figure 8, compared with the control group, the $24 \mathrm{~h}$ drainage, total drainage, chest tube extraction time, hospital stay, and complication rates for patients in the intervention group were greatly reduced, and the differences were statistically observable $(P<0.05)$.

\subsection{The Impacts of Perioperative Nursing Intervention on the} Quality of Life of Patients. The SF-36 scale is a questionnaire developed by the Boston Institute of Health to assess the health status of patients. The SF-36 scale has good reliability and validity, so it has been widely used in the evaluation of

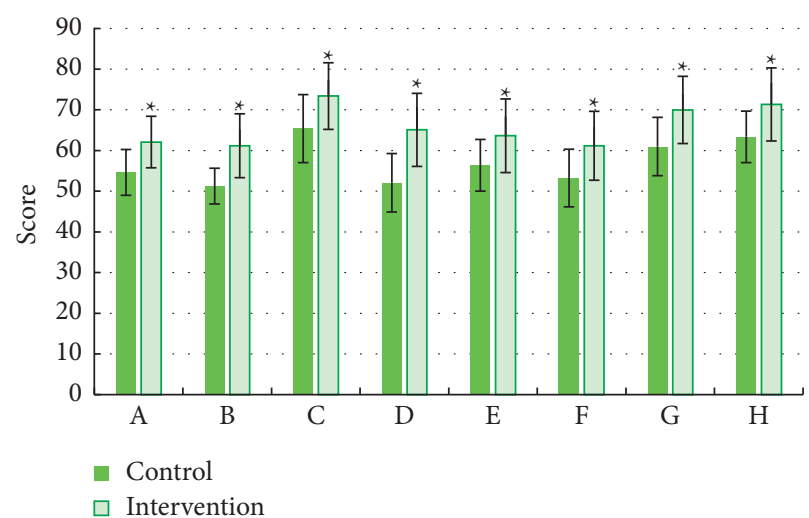

Figure 9: Comparison of differences in quality-of-life scores between the two groups of patients after nursing intervention. (a-h) in the figure referred to the 8 dimensions of the SF-36 scale; * the difference was statistically obvious in contrast to the control group $(P<0.05)$.

the quality of life of the general population and the evaluation of the effect of clinical trials [24]. Therefore, the SF-36 scale was applied to evaluate and compare the differences in the quality of life scores between the two groups of patients after treatment in this study. The results given in Figure 9 suggested that in contrast to the control group, the scores of all dimensions of the quality of life of the intervention group increased remarkably, and the differences were statistically obvious $(P<0.05)$.

\section{Discussion}

The incidence of lung cancer continues to increase, which is a serious threat to human health. Surgical treatment of lung 
cancer betters the patient's physical condition and improves the quality of life of the patient. The perioperative care of the patient after the operation is of great significance for the smooth completion of the operation and the guarantee of the treatment effect $[25,26]$. CT examination plays an important role in the diagnosis of lung cancer, and the misdiagnosis phenomenon in it cannot be ignored. CT manifestations of lung cancer mainly include increased bronchial wall thickness in the lung, bronchial lumen stenosis or obstruction, and concurrent obstructive atelectasis, emphysema. Peripheral lung cancer is usually isolated masses, lobes, and spinous processes. The signs of lobes are unbalanced growth and restriction of peripheral blood vessels and bronchi. The inflation sign of bronchioles is a thin strip with a diameter of about $1 \mathrm{~mm}$, and the air density shadow is about dilated bronchioles. CT scan is also an important method in the diagnosis of lung cancer. CT scans produce a large amount of images. A large number of CT images increase the workload of physicians and therefore increase the probability of misdiagnosis. The combination of imaging computing and deep learning algorithms in image processing, pattern recognition, and artificial intelligence technology provides convenience for CT image denoising, segmentation, and feature extraction and classification [27]. With the rise of multislice spiral computed tomography (MSCT), noninvasive coronary artery imaging technology has been developed. MSCT can improve heart rate artifacts, better display the range of lesions, and identify the nature of plaques. Deep learning shows good results in CT images. Deep learning learns more useful features by constructing a machine learning model with hidden layers and a large amount of training data, thereby improving the accuracy of classification or prediction. Deep learning can portray datarich internal information.

In this study, a 2D CNN and 3D CNN fusion model was constructed and applied to the segmentation processing of $\mathrm{CT}$ images of lung cancer patients to evaluate the segmentation effect and analyze the model qualitatively and quantitatively. The accuracy rate of lung cancer diagnosis was $96.7 \%$. The Dice value (0.876), sensitivity $(0.849)$, and PPV (0.875) of the constructed model for CT image segmentation were higher than those of other models. Yu et al. [28] found that CNN can obtain good accuracy in preprocessing, classification, and segmentation of lung cancer CT images. CT scanning is very important for lung cancer detection. Gayathri Devi and Radhakrishnan [29] proposed to adopt the adaptive threshold technology for air bag segmentation and used a cascaded feedforward neural network based on machine learning. This network enhances the boundary detection algorithm. The proposed neural network performs experiments on CT database images, with an accuracy rate of $98 \%$ and a minimum error rate. This research also shows that intelligent algorithms have a high accuracy rate.

\section{Conclusion}

On the basis of deep learning technology, a 2D CNN and 3D $\mathrm{CNN}$ fusion model was constructed and applied to the segmentation processing of CT images of lung cancer patients to evaluate the segmentation effect and analyze the model qualitatively and quantitatively in this study. The results suggested that the segmentation efficiency of the proposed model was obviously better in contrast to the $2 \mathrm{D}$ $\mathrm{CNN}, 3 \mathrm{D} \mathrm{CNN}$, and U-Net models. Its accuracy in lung cancer diagnosis can be as high as $96.7 \%$. Afterwards, the impacts of perioperative nursing intervention on the patient's postoperative treatment effect were analyzed, and it was found that the implementation of perioperative rehabilitation nursing intervention can effectively improve the pulmonary function, blood gas indexes, and quality of life of patients after surgery. In addition, it can reduce the incidence of postoperative complications and shorten the hospital stay. However, it only analyzed the deep learning model for segmentation processing of lung cancer CT images in this study, so it was necessary to compare and analyze the effect of this model on the effect of surgical treatment after CT image processing in the following studies. In short, the results of this study can provide a reference for improving the diagnosis rate and treatment effect of lung cancer patients.

\section{Data Availability}

The data used to support the findings of this study are available from the corresponding author upon request.

\section{Conflicts of Interest}

The authors declare that they have no conflicts of interest regarding the publication of this paper.

\section{References}

[1] G. A. Rivera and H. Wakelee, "Lung cancer in never smokers," Lung Cancer and Personalized Medicine, vol. 893, pp. 43-57, 2016, PMID: 26667338.

[2] J. Rodriguez-Canales, E. Parra-Cuentas, and II. Wistuba, "Diagnosis and molecular classification of lung cancer," Cancer Treatment and Research, vol. 170, pp. 25-46, 2016, PMID: 27535388.

[3] A. Romaszko and A. Doboszyńska, "Multiple primary lung cancer: a literature review," Advances in Clinical and Experimental Medicine, vol. 27, no. 5, pp. 725-730, 2018, PMID: 29790681.

[4] J. H. Pedersen, Z. Saghir, M. M. Wille, L. H. Thomsen, B. G. Skov, and H. Ashraf, "Ground-glass opacity lung nodules in the era of lung cancer CT screening: radiology, pathology, and clinical management," Oncology, vol. 30, no. 3, pp. 266-274, 2016.

[5] P. Rousset, C. Rousset-Jablonski, M. Alifano, A. MansuetLupo, J.-N. Buy, and M.-P. Revel, "Thoracic endometriosis syndrome: CT and MRI features," Clinical Radiology, vol. 69, no. 3, pp. 323-330, 2014, Epub 2013 Dec 11. PMID: 24331768.

[6] A. Chen, R. A. Karwoski, D. S. Gierada, B. J. Bartholmai, and C. W. Koo, "Quantitative CT analysis of diffuse lung disease," RadioGraphics, vol. 40, no. 1, pp. 28-43, 2020, Epub 2019 Nov 29. PMID: 31782933.

[7] H. W. Goo and J. M. Goo, "Dual-energy CT: new horizon in medical imaging," Korean Journal of Radiology, vol. 18, no. 4, pp. 555-569, 2017, Epub 2017 May 19. PMID: 28670151. 
[8] H. MacMahon, D. P. Naidich, J. M. Goo et al., "Guidelines for management of incidental pulmonary nodules detected on CT images: from the fleischner society 2017," Radiology, vol. 284, no. 1, pp. 228-243, 2017, Epub 2017 Feb 23. PMID: 28240562.

[9] C. Kratochwil, P. Flechsig, T. Lindner et al., "68Ga-FAPI PET/ CT: tracer uptake in 28 different kinds of cancer," Journal of Nuclear Medicine, vol. 60, no. 6, pp. 801-805, 2019, Epub 2019 Apr 6. PMID: 30954939.

[10] X. Li, F. Guo, Z. Zhou et al., "[Performance of deep-learningbased artificial intelligence on detection of pulmonary nodules in chest CT]," Zhongguo Fei Ai Za Zhi, vol. 22, no. 6, pp. 336-340, 2019, Chinese PMID: 31196366.

[11] S. M. Humphries, A. M. Notary, J. P. Centeno et al., "Deep learning enables automatic classification of emphysema pattern at CT," Radiology, vol. 294, no. 2, pp. 434-444, 2020, Epub 2019 Dec 3. PMID: 31793851; PMCID: PMC6996603.

[12] M. Avanzo, J. Stancanello, G. Pirrone, and G. Sartor, "Radiomics and deep learning in lung cancer," Strahlentherapie und Onkologie, vol. 196, no. 10, pp. 879-887, 2020, Epub 2020 May 4. PMID: 32367456.

[13] L. Chen, P. Bentley, K. Mori, K. Misawa, M. Fujiwara, and D. Rueckert, "DRINet for medical image segmentation," IEEE Transactions on Medical Imaging, vol. 37, no. 11, pp. 24532462, 2018, Epub 2018 May 10. PMID: 29993738.

[14] F. P. An and Z. W. Liu, "Medical image segmentation algorithm based on feedback mechanism CNN," Contrast Media and Molecular Imaging, vol. 2019, Article ID 6134942, 13 pages, 2019.

[15] S. Zheng, X. Lin, W. Zhang et al., "MDCC-Net: multiscale double-channel convolution U-Net framework for colorectal tumor segmentation," Computers in Biology and Medicine, vol. 130, 2021 Epub 2020 Dec 16. PMID: 33360107, Article ID 104183.

[16] Y. Wang, Z. Yu, and L. Zhu, "Foreground detection with deeply learned multi-scale spatial-temporal features," Sensors, vol. 18 , no. 12 , p. 4269, 2018.

[17] K. Munir, H. Elahi, A. Ayub, F. Frezza, and A. Rizzi, "Cancer diagnosis using deep learning: a bibliographic review," Cancers, vol. 11, no. 9, p. 1235, 2019.

[18] J. Yu, B. Yang, J. Wang, J. Leader, D. Wilson, and J. Pu, “2D $\mathrm{CNN}$ versus 3D CNN for false-positive reduction in lung cancer screening," Journal of Medical Imaging, vol. 7, no. 5, Epub 2020 Oct 13. PMID: 33062802, Article ID 051202, 2020.

[19] A. Chaddad, P. Sargos, and C. Desrosiers, "Modeling texture in deep 3D CNN for survival analysis," IEEE Journal of Biomedical and Health Informatics, vol. 25, no. 7, pp. 24542462, 2021, Epub ahead of print. PMID: 32960772.

[20] T. Falk, D. Mai, R. Bensch et al., "U-Net: deep learning for cell counting, detection, and morphometry," Nature Methods, vol. 16, no. 1, pp. 67-70, 2019.

[21] E. Pehlivan, A. Balc1, L. Kılıç, and F. Kadakal, "Preoperative pulmonary rehabilitation for lung transplant: effects on pulmonary function, exercise capacity, and quality of life; first results in Turkey," Exp Clin Transplant, vol. 16, no. 4, pp. 455-460, 2018, Epub 2017 Sep 30. PMID: 28969527.

[22] F. Dogar, M. Argun, S. Erdem, K. Gurbuz, A. S. Argun, and I. H. Kafadar, "Clinical and radiological results of surgically treated patients with adolescent idiopathic scoliosis and the effects of pulmonary rehabilitation on respiration functions," Medicine, vol. 100, no. 7, p. e24675, 2021 PMID: 33607805; PMCID: PMC7899867, Article ID e24675.

[23] A. R. Bain, I. Drvis, Z. Dujic, D. B. MacLeod, and P. N. Ainslie, "Physiology of static breath holding in elite apneists," Experimental Physiology, vol. 103, no. 5, pp. 635-651, 2018.
[24] L. Lins and F. M. Carvalho, "SF-36 total score as a single measure of health-related quality of life: scoping review," SAGE Open Medicine, vol. 4, Article ID 2050312116671725, 2016.

[25] B. C. Brocki, E. Westerdahl, D. Langer, D. S. R. Souza, and J. J. Andreasen, "Decrease in pulmonary function and oxygenation after lung resection," ERJ Open Research, vol. 4, no. 1, pp. 00055-02017, 2018, PMID: 29362707; PMCID: PMC5773813.

[26] L. Fernández-Rodríguez, I. Torres, D. Romera et al., "Prediction of postoperative lung function after major lung resection for lung cancer using volumetric computed tomography," The Journal of Thoracic and Cardiovascular Surgery, vol. 156, no. 6, pp. 2297-2308, 2018, Epub 2018 Aug 2. PMID: 30195604.

[27] D. Blanc, V. Racine, A. Khalil et al., "Artificial intelligence solution to classify pulmonary nodules on CT," Diagnostic and Interventional Imaging, vol. 101, no. 12, pp. 803-810, 2020, Epub 2020 Nov 7. PMID: 33168496.

[28] K.-H. Yu, T.-L. M. Lee, M.-H. Yen et al., "Reproducible machine learning methods for lung cancer detection using computed tomography images: algorithm development and validation," Journal of Medical Internet Research, vol. 22, no. 8, PMID: 32755895, Article ID e16709, 2020.

[29] K. Gayathri Devi and R. Radhakrishnan, "Automatic segmentation of colon in 3D CT images and removal of opacified fluid using cascade feed forward neural network," Computational and Mathematical Methods in Medicine, vol. 201515 pages, 2015, Epub 2015 Mar 9. PMID: 25838838; PMCID: PMC4369940, Article ID 670739. 